\title{
Case Report: A case of immune checkpoint inhibitor therapy in a patient with multiple sclerosis [version 1; peer review: 1
}

\section{not approved]}

\author{
Raju Vaddepally (iD1, Soujanya Sodavarapu², Anupama Kutadi³, Wesley Taylor, \\ Navneeth Kumar ${ }^{4}$ \\ ${ }^{1}$ Hematology/Medical Oncology Department, Yuma Regional Medical Center, Yuma, AZ, 85364, USA \\ 2Internal Medicine Department, San Joaquin General Hospital, French Camp, CA, 95231, USA \\ ${ }^{3}$ Pediatrics Department, Yuma Regional Medical Center, Yuma, AZ, 85364, USA \\ ${ }^{4}$ Family Medicine Department, Yuma Regional Medical Center, Yuma, AZ, 85364, USA
}

V1 First published: 23 Sep 2020, 9:1167

https://doi.org/10.12688/f1000research.25490.1

Latest published: 23 Sep 2020, 9:1167

https://doi.org/10.12688/f1000research.25490.1

\section{Abstract}

Immune checkpoint inhibitors (ICIs) have rapidly shifted the landscape of treatments in malignancy with significant improvements in survival paradigm. They have been an attractive armamentarium to the oncologists given the limited immune adverse effects with potential for deeper and durable benefits that haven't been previously noticed with chemotherapy. However, they result in unique toxicities by limiting immune self-tolerance and cause immune-mediated endocrinopathies, such as hypothyroidism, pneumonitis, colitis, hepatitis, myocarditis, meningitis, hypophysitis, etc. As such, they are contraindicated in patients with autoimmune disorders or recipients of organ transplants given the risk for reactivation or flare of the underlying autoimmune disease and rejection of the donor organ in transplants, although sporadic cases have been reported with the use of immunotherapy in such patients. Malignant melanoma is a highly aggressive cancer, with only $15-20 \%$ five-year survival rate once it has spread to the lymph nodes or has distant metastasis. ICIs have changed the landscape of advanced melanoma with exponential improvements in survival, the 5-year survival rates are about $50 \%$. Multiple sclerosis (MS) is recognized as T cell-mediated immune response causing inflammation, which causes local inflammatory plaques and demyelination. ICIs are likely to generate an immune response that causes molecular mimicry and cross-react with CNS autoantigens, in turn exacerbating pre-existing immune response and subsequent flare-ups in MS. There is little knowledge about treating such patients with immunotherapy, short of a few case reports and series; in this report, we describe another such case. We present a case of checkpoint inhibitor therapy in a patient with multiple sclerosis who underwent immune checkpoint inhibitor therapy with

\section{Peer review discontinued \\ Peer review at F1000Research is author- driven. Currently no reviewers are being invited. What does this mean?}

\begin{tabular}{lc} 
Approval Status $\times$ & 1 \\
\hline version 1 & $\times$ \\
23 Sep 2020 & view
\end{tabular}

1. Angela Vidal-Jordana, Multiple Sclerosis Centre of Catalonia, Edifici Cemcat, Hospital Universitari Vall d'Hebron, Universitat

Autònoma de Barcelona, Barcelona, Spain

Any reports and responses or comments on the article can be found at the end of the article. 
pembrolizumab for metastatic malignant melanoma who had a complete response to treatment at the cost of MS relapse, which was managed with high-dose steroids.

\section{Keywords}

Immunotherapy, checkpoint inhibitors, pembrolizumab, malignant

melanoma, multiple sclerosis, malignancy, antigens, toxicites, adverse effects, steroids

\section{Corresponding author: Raju Vaddepally (rvaddepally@yumaregional.org)}

Author roles: Vaddepally R: Conceptualization, Formal Analysis, Funding Acquisition, Supervision, Writing - Original Draft Preparation, Writing - Review \& Editing; Sodavarapu S: Resources, Writing - Original Draft Preparation; Kutadi A: Supervision, Visualization, Writing Original Draft Preparation; Taylor W: Resources; Kumar N: Resources, Writing - Original Draft Preparation

Competing interests: No competing interests were disclosed.

Grant information: This work was supported by the McDaniel Grant - community foundation at Yuma Regional Medical Center. The funders had no role in study design, data collection and analysis, decision to publish, or preparation of the manuscript.

Copyright: (c) 2020 Vaddepally R et al. This is an open access article distributed under the terms of the Creative Commons Attribution License, which permits unrestricted use, distribution, and reproduction in any medium, provided the original work is properly cited.

How to cite this article: Vaddepally R, Sodavarapu S, Kutadi A et al. Case Report: A case of immune checkpoint inhibitor therapy in a patient with multiple sclerosis [version 1; peer review: 1 not approved] F1000Research 2020, 9:1167

https://doi.org/10.12688/f1000research.25490.1

First published: 23 Sep 2020, 9:1167 https://doi.org/10.12688/f1000research.25490.1 


\section{Introduction}

Immune checkpoint inhibitor therapy has transformed the cancer care landscape ${ }^{1}$. It is being used to treat various malignancies with significant improvement in survival noted in solid tumors such as melanoma and non-small cell lung carcinoma, etc $^{2}$. This has been an attractive alternative for many oncologists given its efficacy and minimal toxicity across the spectrum of program death ligand-1 (PDL-1) and program death-1 (PD-1) inhibitors ${ }^{3}$. However, they result in unique toxicities related to immune modulation and activation such as immune-mediated endocrinopathies such as hypothyroidism, pneumonitis, colitis, hepatitis which are seemingly more common compared to rare complications such as myocarditis, meningitis, hypophysitis, etc $^{4}$. As such, checkpoint inhibitor therapy is contraindicated in patients with autoimmune disorders or recipients of organ transplants given the risk for reactivation or flare of the underlying autoimmune disease and rejection of the donor organ in transplants, although sporadic cases have been reported with the use of immunotherapy in such patients ${ }^{5}$. In this report, we present a case of checkpoint inhibitor therapy in a patient with multiple sclerosis (MS) who underwent immune checkpoint inhibitor therapy with pembrolizumab for metastatic malignant melanoma.

\section{Case Report}

A 73-year-old female with a past medical history of MS with chronic right hemi-somal deficits on baclofen $(10 \mathrm{mg}$ twice daily), paroxetine (40 mg daily), and gabapentin (300 mg three times daily). She had prior history of cutaneous malignant melanoma (MM) in 2015 post excision and negative sentinel lymph nodes noticed left inguinal swelling before November in 2018 and a PET scan revealed prominent and bulky metabolic adenopathy involving the left inguinal, external iliac, proximal internal iliac regions, and common iliac region lymphadenopathy (Figure 1 and Figure 2). Left inguinal mass biopsy revealed metastatic malignant melanoma (Figure 3 and Figure 4). Tissue from the biopsy tested positive for HMB-45 and Melan-A antibodies (Figure 5 and Figure 6). Biomarker testing revealed no evidence of BRAF or KIT mutations; TERT, NRAS mutations and MDM2 copy gain was present, MSI-stable, and the TMB high at 8.8 mutation/MB (87th percentile). Magnetic resonance imaging (MRI) of the brain was negative for metastatic malignancy however showed non-specific white matter changes consistent with demyelinating plaques linked to her history of multiple sclerosis without finding of active disease (Figure 7 and Figure 8).

After discussing the pros and cons related to immune checkpoint inhibitors (ICI) versus salvage chemotherapy; she expressed understanding of the potential but significant risk of MS flareup. She was initiated on pembrolizumab $200 \mathrm{mg}$ every 3 weeks at 4 weeks after diagnosis. Unfortunately, two weeks after the first cycle of pembrolizumab therapy she encountered significant lethargy, slurred speech, word-finding difficulty, stiffness and pain in her bilateral groins, gait imbalance with recurrent falls, generalized weakness requiring assistance for bed transfer.

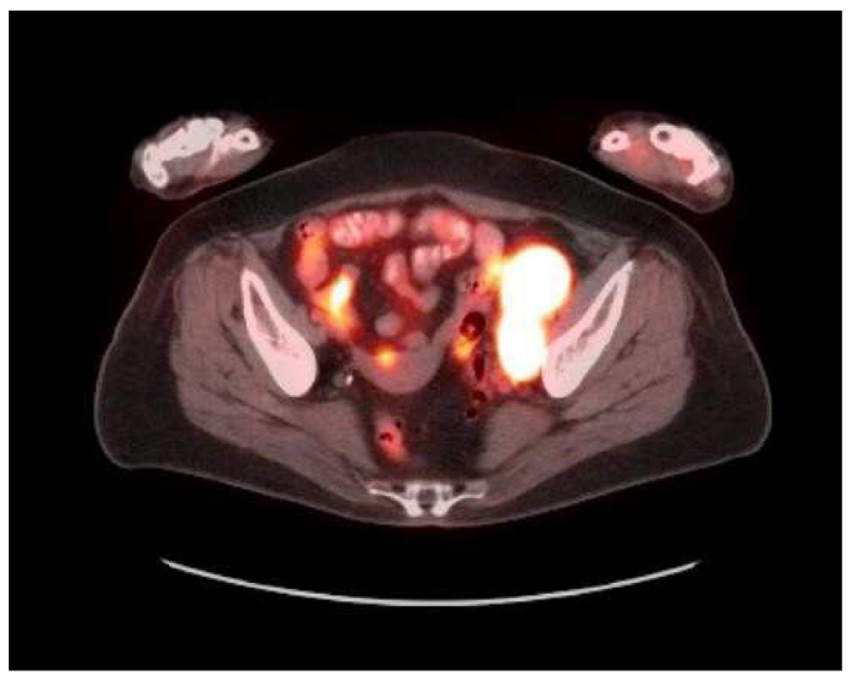

Figure 1. Large prominently metabolic adjacent nodes are visualized in the left inguinal region, measured together these are $2.8 \times 5.6 \mathrm{~cm}$ with an SUV max of 21.1 on image 271 . Multiple prominently metabolic nodes are visualized throughout the left external iliac, proximal internal iliac and common iliac regions. 1 of the more prominent nodes situated in the distal external iliac chain spanning $2.8 \times 4.2 \mathrm{~cm}$ with an SUV max of 18.4 on image 250. Small metabolic nodes are also visualized in the lower retroperitoneum up to the L4 level.

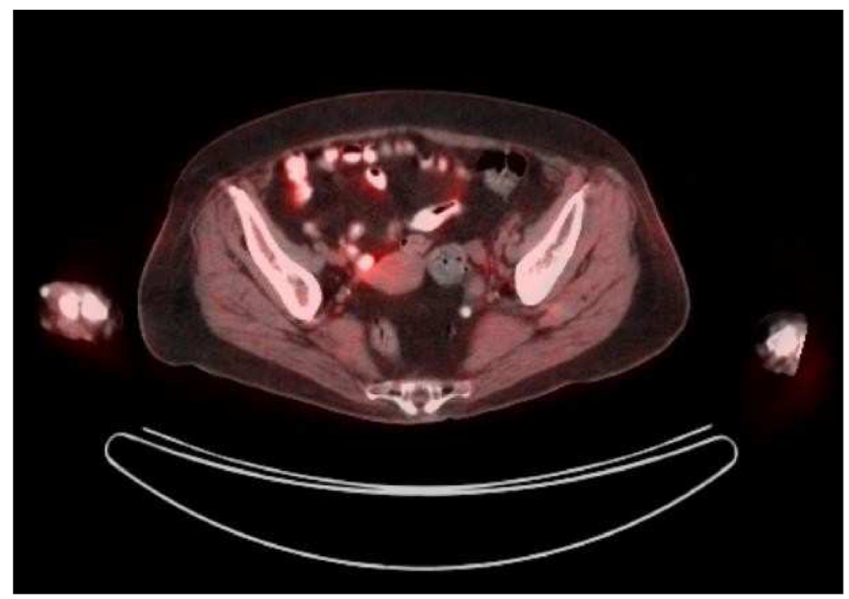

Figure 2. There has been significant interval improvement in previously noted metabolic adenopathy involving the left inguinal region, left pelvis and lower retroperitoneum, with these nodes either entirely normalized or now significantly smaller and no longer appreciably metabolic of background. For example, a left inguinal node is $1.3 \times 1.8 \mathrm{~cm}$ with an SUV max of 1.0; previously a nodal conglomerate in this region was $2.8 \times 5.6 \mathrm{~cm}$ and SUV max 21.1. A small residual nodal prominence in the left external iliac region is $0.9 \times 2.7$ $\mathrm{cm}$, SUV max 2.0; previously 2 adjacent nodes in this region or $3.3 \times 7.6 \mathrm{~cm}$ and SUV max 19.1. No new metabolic nodal lesions are visualized in the inguinal regions, pelvis or throughout the abdomen 


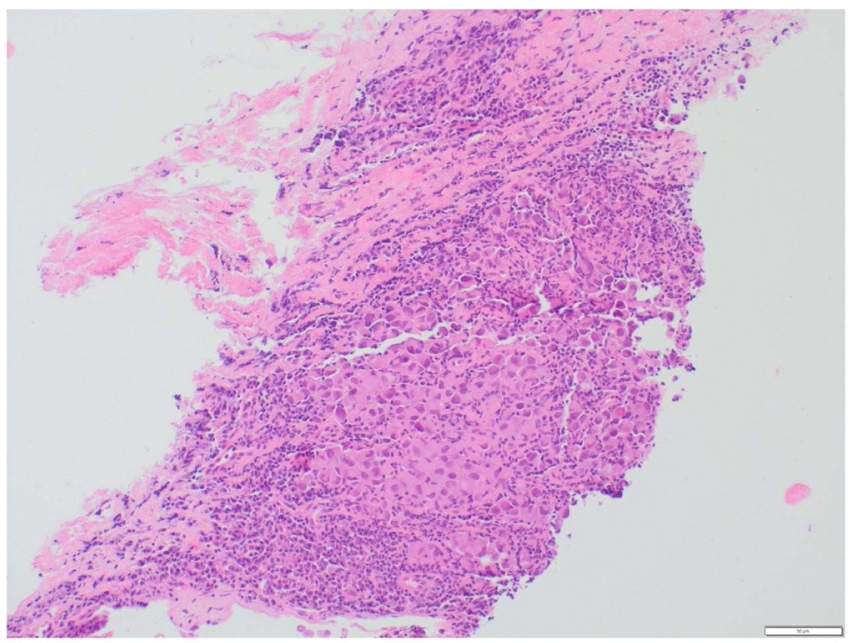

Figure 3. Fragment of lymph node that is involved with metastatic melanoma with hematoxylin-eosin stain (larger pink looking cells). In the background you can see some normal lymphocytes (smaller dark blue staining cells). Magnification, ×20.

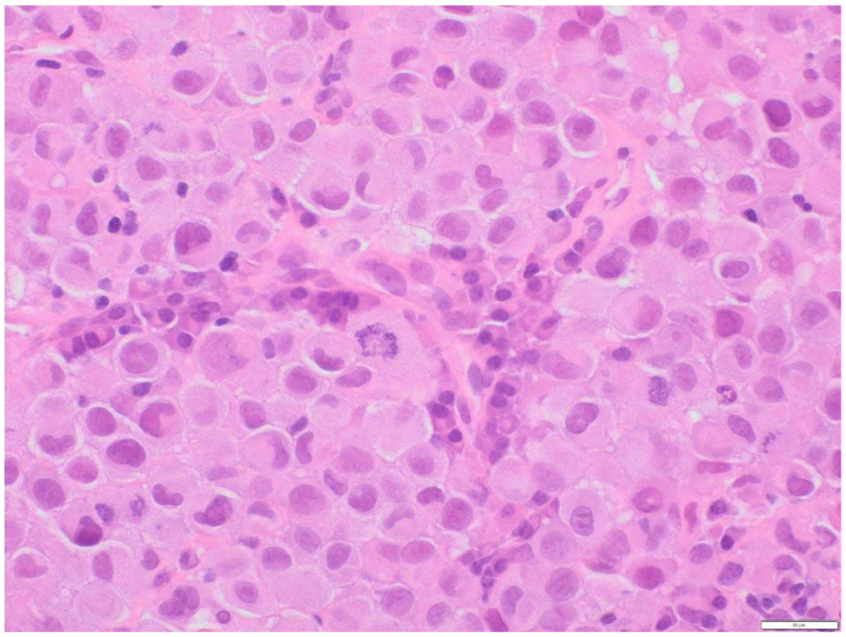

Figure 4. Hematoxylin-eosin stain high-power view of metastatic melanocytes. There are few abnormal mitotic figures and some plasma cells in the background.

Pertinent physical exam findings included pupils that were reactive to light both directly and consensually; the fundoscopic exam was normal with the cup to disc ration, no edema/ hemorrhages; apraxia, tremors in bilateral hands; decreased motor strength ( $4 / 5$ using the Oxford scale) in the bilateral upper and lower extremity muscle groups; deep tendon reflexes $2 / 4$ in the left and $3 / 4$ in the right biceps, triceps, patellar and achilles. Hoffman, Tromner's, and Babinski signs were present on the right; sustained clonus was noted in right ankle and ataxia was present. Complete blood picture, electrolytes, kidney, and liver function were essentially unremarkable. Her signs and symptoms were concerning for an MS flare-up, and an MRI

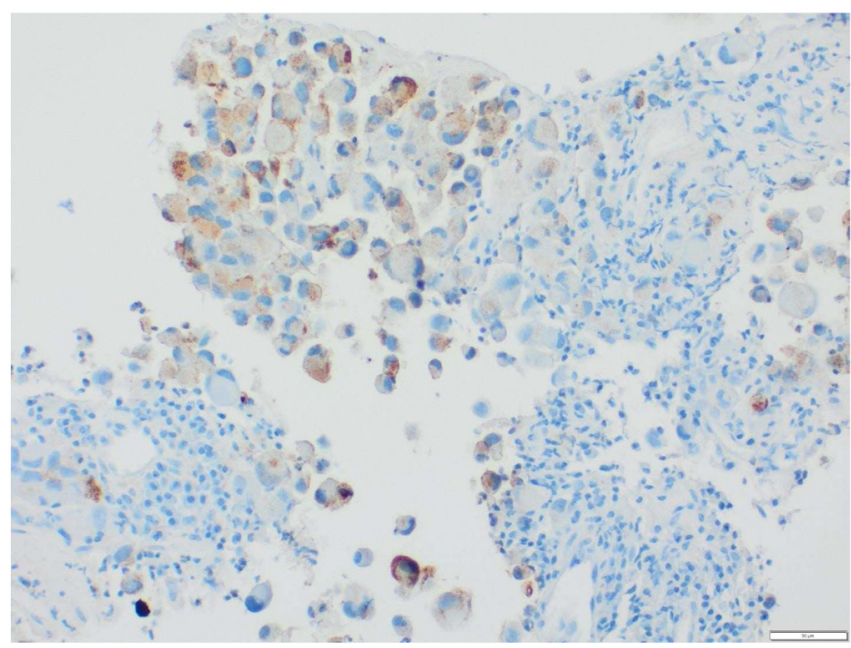

Figure 5. Immunohistochemical stains; HMB45 positive.

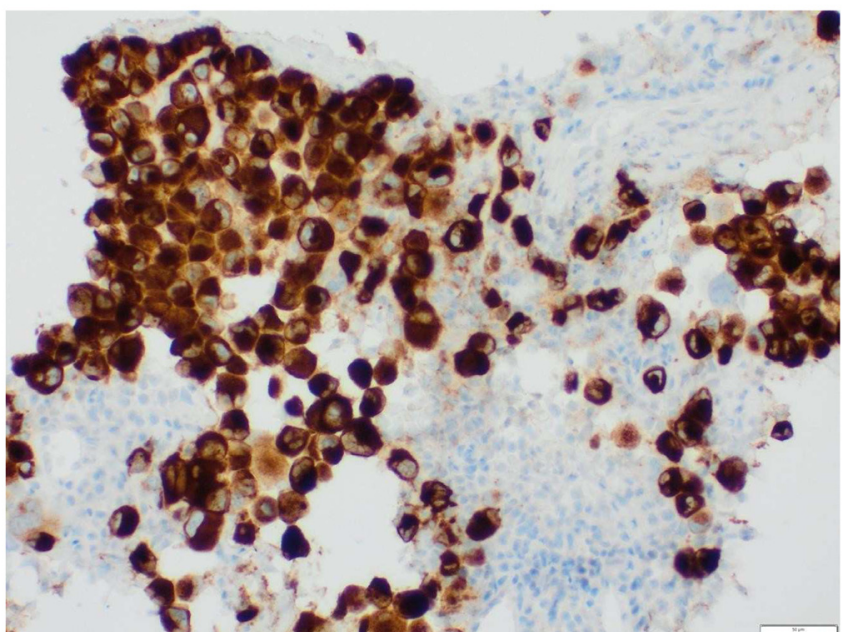

Figure 6. Melan-A positive indicating melanocyte origin with brown cytoplasmic staining.

of the brain with and without contrast revealed enhancing white matter lesion on the right external capsule, larger in size and hyperintense per the gadolinium contrast; EEG was unremarkable. In light of clinical and radiological findings consistent with an MS flare-up, she was started on prednisone $1 \mathrm{mg} / \mathrm{kg}$ with a prolonged taper over 8 weeks by $10 \mathrm{mg}$ every week with subsequent significant improvement in treatment-related neurological symptoms in the coming weeks with active participation in physical therapy (PT).

She had an interval improvement in the tumor size with just one round of treatment, which has been correlated in studies with patients encountering immune-related adverse effects (iAE's) often having better responses than their counterparts who do not have iAE's ${ }^{6}$. CT imaging of the abdomen/pelvis with contrast imaging post-cycle showed significant interval response 


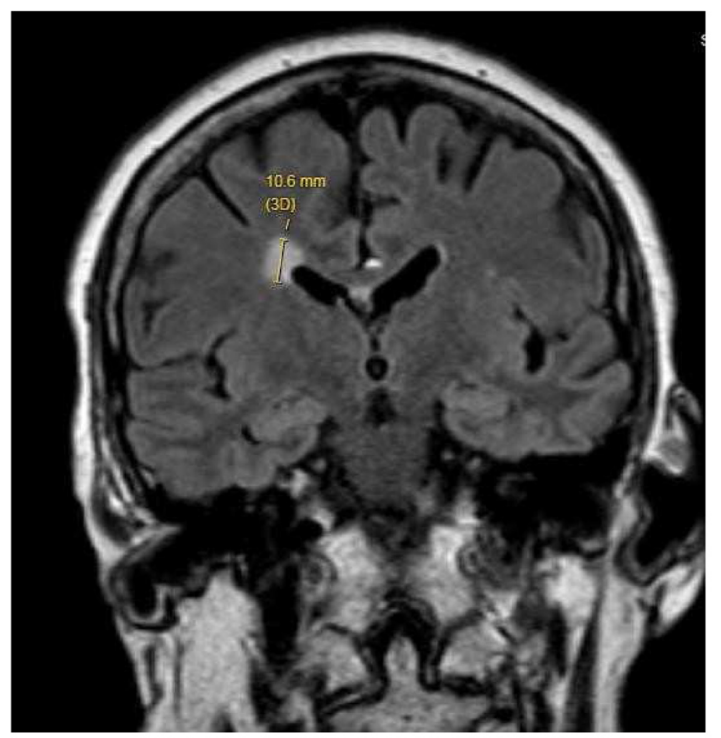

Figure 7. Coronal MRI brain showing $\mathbf{1 0 . 1} \mathbf{~ m m}$. Nonspecific focal white matter lesions could reflect multiple sclerosis, without finding of active disease.

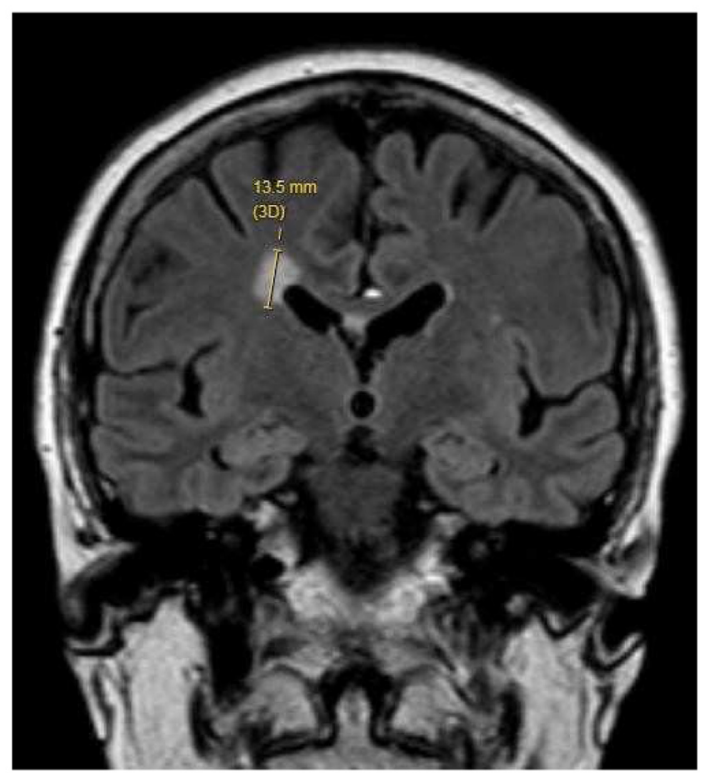

Figure 8. Enhancing white matter lesion on the right external capsule, larger in size as well.

with the adenopathy decreased by $>50 \%$, for example, left inguinal lymph node decreased from 4.6 to $1.3 \mathrm{~cm}$, left external iliac adenopathy decreased from 4.7 to $2.1 \mathrm{~cm}$, and the para-aortic lymph node is essentially resolved, measuring $4 \mathrm{~mm}$ where it previously measured $11 \mathrm{~mm}$. Two months into her complete convalescence from neurological adverse effects related to the MS flare-up, she was rechallenged with a second round of pembrolizumab therapy with a baseline of continued prednisone $20 \mathrm{mg}$, which she tolerated well without MS relapse.
She subsequently encountered immune-mediated acute hepatitis and recurrent MS flare-up after cycle 4 of therapy. Labs revealed liver transaminases elevated to 20 times upper limit of normal (ULN)- AST 711 U/L, ALT 978 U/L, and ALP 392 U/L ( $3 \times$ ULN) with normal total bilirubin $1.1 \mathrm{mg} / \mathrm{dl}$. She was then subjected to another round of high-dose prednisone $1.5 \mathrm{mg} / \mathrm{kg}$ with a prolonged taper, requiring a stay at a skilled nursing facility and PT for around 1 month. Restaging PET scan after four cycles of immunotherapy in July 2019 revealed significant interval improvement: previous prominent metabolic adenopathy in the left pelvis/inguinal region and lower retroperitoneum had completely resolved. Small residual nodal lesions remained in the left inguinal region and left external iliac chain, with these demonstrating only low level/background metabolic activity. For example, a left inguinal node was $1.3 \times 1.8 \mathrm{~cm}$ with an SUV max of 1.0 on axial image 271 - previously a nodal conglomerate in this region was $2.8 \times 5.6 \mathrm{~cm}$ and SUV max 21.1; a small residual nodal prominence in the left external iliac region was $0.9 \times 2.7 \mathrm{~cm}, \quad$ SUV $\max 2.0$ on image 256, whereas previously two adjacent nodes in this region were $3.3 \times 7.6 \mathrm{~cm}$ and SUV max 19.1. At this point, given recurrent and severe CPI-mediated immune-related adverse effects with MS flare-ups and hepatitis, further immunotherapy was discontinued. The patient continued to exhibit an incomplete response thereafter, but with decreased quality of life given the persisting debility from MS relapse.

\section{Discussion}

Malignant melanoma is a highly aggressive cancer, with only a $15-20 \%$ five-year survival rate once it has spread to the lymph nodes or has distant metastasis? ${ }^{7}$. The development of ICIs has changed the face of melanoma treatment and has improved the survival rates of these patients. The drugs in this class have been directed against CTLA-4, PD-1 and PD-L1, namely ipilimumab, nivolumab and pembrolizumab, respectively ${ }^{3}$. ICIs have changed the landscape of advanced melanoma with exponential improvements in survival, the 5-year survival rates were about $50 \%{ }^{8,9}$

ICIs can present with a wide range of irAEs due to lack of selectivity and by their generalized immune reaction ${ }^{10}$. These effects range from systemic autoimmune complications to severe neurological complications ${ }^{11}$. The neurological complications have been estimated to have an incidence of $2-4 \%$ with severe neurological manifestations, including MS, being reported in $0.2-0.4 \%$ of patients treated with pembrolizumab and nivolumab $^{11}$.

MS is recognized as $\mathrm{T}$ cell-mediated immune response causing inflammation, which causes local inflammatory plaques and demyelination ${ }^{7}$. ICIs are likely to generate an immune response that causes molecular mimicry and cross-react with CNS autoantigens, in turn exacerbating pre-existing immune response and subsequent flare-ups in $\mathrm{MS}^{11,12}$.

On an extensive literature review, we could come across very few reports of ICIs use in patients with MS. Isitan et al. described a 46-year-old with stage IV metastatic non-small cell 
lung cancer who was treated with one dose of atezolizumab, who subsequently developed relapse of MS with worsening features and refractory to treatment ${ }^{13}$. Another case, described by Gettings et al., was of a 56-year-old male with MS with recurrent melanoma after multiple resections; he was treated with ipilimumab who developed two MS flare-ups, leaving him with disability from MS relapse but had remission of melanoma on PET scan ${ }^{14}$. Garcia et al. conducted a retrospective analysis capturing 42,529 adverse events with the use of ICI with 13 cases identified with MS. Of the 13, eight had a previous diagnosis of MS. All the patients in their analysis experienced progression of their MS, and two patients died following their MS relapse ${ }^{15}$.

Our patient had her first MS relapse after the first dose of pembrolizumab, which resolved with high-dose steroids. She did not develop any flare-ups with the second and third rounds of therapy, but again encountered a relapse during the fourth round, which was again successfully treated with steroids but with delayed convalescence. At the end of the fourth round patient achieved complete remission of metastatic malignant melanoma. Our case adds to the pool of existing clinical data that immune checkpoint inhibitors are universally detrimental to patients with MS, given definitive relapse identified in almost all the patients identified during the literature review and our personal experience.

\section{Data availability}

All data underlying the results are available as part of the article and no additional source data are required.

\section{Consent}

Written informed consent for publication of their clinical details and clinical images was obtained from the patient.

\section{References}

1. Havel $\|$, Chowell D, Chan TA: The evolving landscape of biomarkers for checkpoint inhibitor immunotherapy. Nat Rev Cancer. 2019; 19(3): 133-150.

PubMed Abstract | Publisher Full Text | Free Full Text

2. Youn B, Trikalinos NA, Mor V, et al:: Real-world use and survival outcomes of immune checkpoint inhibitors in older adults with non-small cell lung cancer. Cancer. 2020; 126(5): 978-985.

PubMed Abstract | Publisher Full Text

3. Vaddepally RK, Kharel P, Pandey R, et al.: Review of Indications of FDAApproved Immune Checkpoint Inhibitors per NCCN Guidelines with the Level of Evidence. Cancers (Basel). 2020; 12(3): 738 PubMed Abstract | Publisher Full Text | Free Full Text

4. Kumar V, Chaudhary N, Garg M, et al.: Current Diagnosis and Management of Immune Related Adverse Events (irAEs) Induced by Immune Checkpoint Inhibitor Therapy. Front Pharmacol. 2017; 8: 49 PubMed Abstract | Publisher Full Text | Free Full Text

5. Johnson DB, Sullivan RJ, Menzies AM: Immune checkpoint inhibitors in challenging populations. Cancer. 2017; 123(11): 1904-1911. PubMed Abstract | Publisher Full Text | Free Full Text

6. Prior LM, Harrold E, O'Leary CG, et al.: Toxicities in immunotherapy: Can they predict response? J Clin Oncol. 2016; 34(15 suppl): e14534. Publisher Full Text

7. Weiss SA, Hanniford D, Hernando E, et al:: Revisiting determinants of prognosis in cutaneous melanoma. Cancer. 2015; 121(23): 4108-4123. PubMed Abstract | Publisher Full Text | Free Full Text

8. Robert C, Ribas A, Schachter J, et al.: Pembrolizumab versus ipilimumab in advanced melanoma (KEYNOTE-006): post-hoc 5-year results from an open-label, multicentre, randomised, controlled, phase 3 study. Lancet
Oncol. 2019; 20(9): 1239-1251.

PubMed Abstract | Publisher Full Text

9. Larkin J, Chiarion-Sileni V, Gonzalez R, et al.: Combined Nivolumab and Ipilimumab or Monotherapy in Untreated Melanoma. N Engl J Med. 2015; 373(1): 23-34

PubMed Abstract | Publisher Full Text | Free Full Text

10. Yshii LM, Hohlfeld R, Liblau RS: Inflammatory CNS disease caused by immune checkpoint inhibitors: status and perspectives. Nat Rev Neurol. 2017; 13(12): 755-763.

PubMed Abstract | Publisher Full Text

11. Dalakas MC: Neurological complications of immune checkpoint inhibitors: what happens when you 'take the brakes off' the immune system. Ther Adv Neurol Disord. 2018; 11: 1756286418799864

PubMed Abstract | Publisher Full Text | Free Full Text

12. Huang WJ, Chen WW, Zhang X: Multiple sclerosis: Pathology, diagnosis and treatments. Exp Ther Med. 2017; 13(6): 3163-3166. PubMed Abstract | Publisher Full Text | Free Full Text

13. Isitan C, Wesley S: Safety of Checkpoint Inhibitors for Cancer Treatment in Patients with Multiple Sclerosis: A Case Report (P1.7-006). Neurology. 2019; 92(15 Supplement): P1.7-006 Reference Source

14. Gettings EJ, Hackett CT, Scott TF: Severe relapse in a multiple sclerosis patient associated with ipilimumab treatment of melanoma. Mult Scler. 2015; 21(5): 670 . 2015; 21(5): 670.
PubMed Abstract | Publisher Full Text

15. Garcia $C R$, Jayswal $R$, Adams $V$, et al.: Multiple sclerosis outcomes after cancer immunotherapy. Clin Transl Oncol. 2019; 21(10): 1336-1342. PubMed Abstract | Publisher Full Text | Free Full Text 


\section{Peer review discontinued peer review at F100oResearch is author-driven. Currently no reviewers are being invited. What does this mean?}

\section{Version 1}

Reviewer Report 02 February 2021

https://doi.org/10.5256/f1000research.28129.r76659

(C) 2021 Vidal-Jordana A. This is an open access peer review report distributed under the terms of the Creative Commons Attribution License, which permits unrestricted use, distribution, and reproduction in any medium, provided the original work is properly cited.

\section{Angela Vidal-Jordana}

Department of Neurology-Neuroimmunology, Multiple Sclerosis Centre of Catalonia, Edifici Cemcat, Hospital Universitari Vall d'Hebron, Universitat Autònoma de Barcelona, Barcelona, Spain

The authors use different terms regarding Multiple Sclerosis disease that are not correct and/or not commonly used in the literature. Some of them are listed below:

In the abstract and the discussion section, they state that MS is recognized as a T cellmediated immune disease. With the advent of the new therapies targeting B cells (such as ocrelizumab, rituximab, ofatumumab, among others) this statement has been questioned and does not hold true anymore.

They use the term "flare-ups" where they should use the more correct term "relapses" when referring to neurological worsening due to MS.

In the case report section, page 3, paragraph 2 the authors state: “MRI (....) showed nonspecific white matter changes consistent with demyelinating plaques linked to her history of MS without finding of active disease". White matter lesions suggestive of MS are not considered non-specific, in fact, they should meet some specific criteria in order to attribute the lesions to MS, not only in shape but also in localization. Also, I recommend the authors to better specify the meaning of "active disease". Do they refer to new T2 lesions compared to a previous MRI or to Gd-enhancing lesions?

Apart from these terminology issues, my main concern is that the neurological symptoms that the patient presented (lethargy, slurred speech, word-finding difficulty, stiffness, pain, gait imbalance, and generalized weakness requiring assistance for transfers) are most probably not related to her MS. Neither the symptoms are typical for an MS relapse (that are also very infrequent in older patients), nor they can be justified by an increase in the size of a previous lesion in the right external capsule, even in the presence of Gd-enhancement, as lesions in this location do not produce the aforementioned symptomatology. It is most likely that the patient might have suffered from ICANS (or other neurotoxicity related to immune checkpoint inhibitors) that usually also improves with corticosteroid treatment. 
Therefore, in my opinion, the case report's interest was to report the evolution of an MS patient receiving immunotherapy and how it impacted her disease. However, having in mind all the stated above, I am afraid I should recommend not to index the case report in its current form.

Competing Interests: No competing interests were disclosed.

Reviewer Expertise: Multiple sclerosis, neuroimmunology

I confirm that I have read this submission and believe that I have an appropriate level of expertise to state that I do not consider it to be of an acceptable scientific standard, for reasons outlined above.

The benefits of publishing with F1000Research:

- Your article is published within days, with no editorial bias

- You can publish traditional articles, null/negative results, case reports, data notes and more

- The peer review process is transparent and collaborative

- Your article is indexed in PubMed after passing peer review

- Dedicated customer support at every stage

For pre-submission enquiries, contact research@f1000.com 